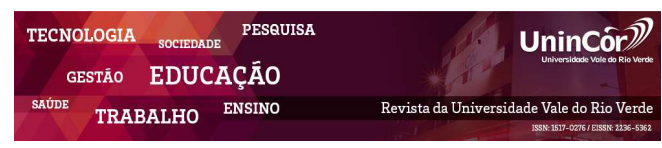

Revista da Universidade Vale do Rio Verde ISSN: 1517-0276 / EISSN: 2236-5362 Vol. 16 | n. 1 | Ano 2018

Rafaella Alves Sarmento Costa Universidade Federal de Campina Grande rafaellasarmento@hotmail.com

Camila Carla Dantas Soares

Universidade Federal de Campina Grande camilacarla.soares@hotmail.com

Jeferson Barbosa Silva Universidade Federal da Paraíba jefersonbarbosa_@hotmail.com

Valkênia Alves Silva

Universidade Estadual da Paraíba kenia3529@gmail.com

Jocelly de Araújo Ferreira Universidade Federal de Campina Grande jocellyaferreira@hotmail.com

\section{A COMUNICAÇÃO ENTRE ENFERMEIROS E PACIENTES CRÍTICOS MASCULINOS: ESTRATÉGIA DE ENFRENTAMENTO}

\section{RESUMO}

A comunicação é caracterizada como um mero instrumento ou meio de difusão e transmissão de informações, no entanto, o profissional de enfermagem, quando consegue harmonizar o cuidado e perceber que a comunicação afetiva e efetiva faz parte deste processo, consegue compreender o pensar, o agir, o sentir, o desejar, o escolher, o valorizar das experiências e permite que o paciente continue a construir sua história de vida. Objetivou-se investigar a importância e os fatores que influenciam na comunicação dos enfermeiros com os pacientes críticos do sexo masculino. Trata-se de uma pesquisa bibliográfica com 23 artigos publicados em periódicos nacionais. O paciente crítico é aquele que possui um determinado grau de comprometimento à saúde, havendo alteração em algumas de suas funções fisiológicas. Os fatores que influenciam na comunicação dos enfermeiros com os pacientes críticos do sexo masculino são a masculinidade, o gênero e a cultura, sendo importante uma comunicação eficaz entre o enfermeiro e o paciente crítico do sexo masculino para humanizar o cuidado dialogando com o paciente para esclarecer os procedimentos realizados, exames diagnósticos, tratamento, ou minimizando suas dúvidas, anseios e angustias causado pela sua condição passiva imposta pela enfermidade e hospitalização. Dessa forma, foi possível observar que através da comunicação novas estratégias sejam elencadas e repassadas aos pacientes, em especial aos do sexo masculino, por ser um público em que é preciso ter mais cautela, saber abordar, dialogar e repassar as informações e os procedimentos que serão realizados.

Palavras-chave: Comunicação. Enfermeiro. Paciente Crítico.

\section{COMMUNICATION BETWEEN NURSES AND CRITICAL MALE PATIENTS: IMPACT STRATEGY}

\begin{abstract}
Communication is characterized as a mere instrument or means of diffusion and transmission of information, however, when the nursing professional can harmonize care and perceive that affective and effective communication is part of this process, he / she can understand thinking, acting, to feel, to desire, to choose, to value the experiences and to allow the patient to continue to build his life history. The objective was to investigate the importance and factors that influence the communication of nurses with critical male patients. This is a bibliographical research with 23 articles published in national journals. The critical patient is
\end{abstract}


one who has a certain degree of commitment to health, and there is a change in some of their physiological functions. The factors influencing nurses' communication with critical male patients are masculinity, gender and culture, and an effective communication between the nurse and the critical male patient is important to humanize care by dialoguing with the patient to clarify the procedures performed, diagnostic tests, treatment, or minimizing their doubts, anxieties and anxieties caused by their passive condition imposed by the illness and hospitalization. In this way, it was possible to observe that through the communication new strategies are listed and passed on to patients, especially males, because it is a public in which one needs to be more cautious, to know how to approach, to dialogue and to pass on the information and procedures that will be performed.

Keywords: Communication. Nurse. Critical Patient.

Recebido em: 20/11/2016 - Aprovado em: 10/01/2018 - Disponibilizado em: 15/07/2018

\section{INTRODUÇÃO}

Nas concepções dominantes, ser homem é tido como ser racional, ativo, provedor, sexualmente irresponsável, poderoso, forte, protetor, violento, decidido, corajoso e invulnerável. Sua masculinidade é idealizada, ao defender a invulnerabilidade e uma sexualidade instintiva, gerando comportamentos pouco saudáveis ou de risco nos homens, e os predispõe, assim, a certas enfermidades, lesões e mortes (COUTO; MACHIN, 2012).

$\mathrm{O}$ alto índice de mortalidade na população masculina indica que os homens, em grande maioria, quando vinculados a questões culturais e educacionais, perpassam por riscos elevados à saúde, como colisões de carro, acidentes de trabalho e uso abusivo de substâncias psicoativas. Os homens procuram os serviços de saúde com menor frequência, e engajam- se menos em comportamentos preventivos (GOMES et al., 2011).

Perante esse estilo de vida, ou seja, com procura reduzida ou demorada aos serviços de saúde, pode resultar em atraso significativo no diagnóstico e no agravamento da morbidade, o que leva a maiores gastos de recursos públicos, além de graves consequências clínicas e econômicas e emocionais para o paciente e sua família (BRASIL, 2009).

Segundo Nascimento et al. (2011), a baixa procura dos homens por práticas preventivas e pouca participação desse público aos serviços de saúde, estão associados a alguns fatores como a pouca valorização do autocuidado, a desatenção com sua saúde e a dificuldade de compartilhar sentimentos e de verbalizar as próprias necessidades. Dessa forma, muitos homens temem que condutas preventivas possam ser interpretadas como demonstração de fraqueza, medo ou de 
insegurança e geram desconfianças acerca da sua masculinidade. Estudos também apontam a preferência dos homens por serviços de saúde de maior complexidade, como hospitais, serviços de urgência e farmácias.

Para Brasil (2009), os homens ao procurarem por serviços de saúde, em especial os voltados para atenção secundária, vem a aumentar o índice de permanência em ambientes hospitalares por estarem muitas vezes em estágio avançado da doença, com condições precárias para uma boa recuperação e cuidados adequados.

A comunicação é caracterizada como um mero instrumento ou meio de difusão e transmissão de informações, no entanto, o profissional de enfermagem, quando consegue harmonizar o cuidado e perceber que a comunicação afetiva e efetiva faz parte deste processo, consegue compreender o pensar, o agir, o sentir, o desejar, o escolher, o valorizar das experiências e permite que o paciente continue a construir sua história de vida, caracterizando a comunicação terapêutica (COSTA, 2009).

Particularmente na área da Enfermagem, é relevante destacar o perfil dessa profissão, presente em seu saber e fazer, nos diversos campos de atuação, respaldado pelo entendimento de que o cuidado envolve interpretação de vivências interpessoais do profissional de saúde com o paciente, sendo muitas vezes necessário o uso da comunicação terapêutica para este entendimento. (SILVA; BRITO, 2010).

Percebe-se a necessidade de mudanças nas estratégias educativas dos estabelecimentos oficiais de assistência à saúde e na capacitação/qualificação contínua de equipes de profissionais, indo além do atendimento a essa parcela da população. Pois as práticas de comunicação e educação em saúde não consideram, em sua maioria, os saberes e fazeres existentes entre os pacientes, sendo marcadas pelo autoritarismo (SCHRAIBER et al., 2010).

Hipotetisando a relevância que a comunicação eficaz entre o enfermeiro e o paciente trás para a recuperação deste, surgiu o interesse de investigar sobre a importância da comunicação dos enfermeiros com os pacientes críticos do sexo masculino e os fatores que influenciam na comunicação dos enfermeiros com os pacientes críticos do sexo masculino. Vários estudos comprovam que a existência de uma boa comunicação onde o indivíduo sente-se livre para expor seus medos, angústias, dúvidas, encontrando conforto e segurança, contribuirá para elevar sua autoestima estimulando o autocuidado e o desejo de recuperação da sua saúde.

\section{MÉTODOS}

Trata-se de uma pesquisa bibliográfica que segundo Marconi e Lakatos 
(2012), pode ser considerada como um estudo formal que utiliza pensamentos reflexivos sobre a temática abordada em textos adquiridos através de artigos, e solicita uma análise científica, constituindo tanto em um caminho para se conhecer a realidade, como na busca por respostas para as questões propostas.

A fim de operacionalizar esse estudo, levou- se em consideração as seguintes etapas: levantamento bibliográfico, análise, interpretação e seleção de material bibliográfico, construção preliminar do estudo e redação final. Este levantamento foi rastreado pela combinação dos descritores: Comunicação; Enfermeiro; Paciente Crítico.

A coleta de dados iniciou-se pela realização de um levantamento bibliográfico acerca da temática, levando em consideração artigos inseridos na base de dados da Scielo (Scientific Electronic Library Onli$\underline{n e}$ ), considerada uma biblioteca eletrônica que abrange uma coleção selecionada de periódicos científicos brasileiros e livros contendo o assunto abordado. Posteriormente foi realizada a análise e a interpretação do material bibliográfico compilado de modo a selecionar os textos relacionados aos objetivos propostos para este estudo. $\mathrm{Na}$ realização da análise foram selecionados 23 artigos, em que sequencialmente foi realizada uma leitura seletiva, a partir da qual foi determinado o material que seria utilizado na pesquisa, selecionando as informações pertinentes à concretude dos objetivos deste estudo.
Após a realização das leituras, elaborou-se um texto de análise dos dados, considerando os objetivos propostos, e configurando-se em: Fatores que influenciam na comunicação dos enfermeiros com os pacientes críticos do sexo masculino; A importância de uma comunicação eficaz entre o enfermeiro e o paciente crítico do sexo masculino.

\section{RESULTADOS E DISCUSSÃO}

\section{Fatores que influenciam na comunicação dos enfermeiros com os pacientes críticos do sexo masculino}

As relações entre masculinidade e cuidado em saúde têm sido analisadas com base na perspectiva de gênero, focalizando as dificuldades dos usuários na busca por assistência de saúde e as formas como os serviços lidam com as dificuldades e demandas específicas do público masculino (COUTO et al, 2010).

Alguns estudos qualitativos identificam barreiras para a presença dos homens nos serviços de saúde, principalmente em relação à busca por assistência à saúde e às representações masculinas de saúde-adoecimento. Para Gomes e Nascimento (2006), as dificuldades dos homens estão diretamente ligadas a estrutura de identidade de gênero, masculinidade, interligando a noção de invulnerabilidade, a busca de risco como um valor na qual dificultaria a verbalização 
de suas necessidades de saúde no contexto da assistência e com os profissionais do sexo oposto.

Gênero é aqui entendido como condições que histórica e socialmente constroem e estabelecem as relações sociais de sexo, ligadas pelo poder e desigualdade, sendo, portanto, um princípio ordenador e normatizador de práticas sociais.

As práticas de cuidar de si e dos outros são construídas nas relações entre as pessoas, tanto em lócus privado/doméstico quanto em público/institucional, ampliar o reconhecimento e o acolhimento de demandas e necessidades masculinas, reforçaria a ruptura do círculo vicioso de invisibilidade e exclusão dos sujeitos, o que permitiria resgatar a equidade e aprimorar o cuidado e a assistência prestada pelos enfermeiros ao público masculino (COUTO et al, 2010).

Corroborando com a organização e rotina dos serviços, pesquisas apontam que as instituições de saúde têm uma influência importante na (re) produção do imaginário social de gênero que, por sua vez, tem repercussões diretas na atenção oferecida à população masculina. Para Courtenay (2000), os serviços de saúde destinam menos tempo de seus profissionais aos homens e oferecem poucas e breves explicações sobre mudanças de fatores de risco para enfermidades aos homens quando comparado com as mulheres. Essas ações reforçam os padrões sociais de masculinidade e feminilidade associados às noções de assistência em saúde.

De acordo com Silveira e Barbosa (2009), a sociedade moderna ainda trás como característica importante à cultura sexual marcada pelo machismo e pela questão do gênero, o que implica em estabelecer relações de poder entre homens e mulheres, trazendo o domínio da masculinidade em detrimento à submissão da feminilidade. E, mais uma vez, se recorre à diferença sexual de gênero para caracterizar os sujeitos, suas demandas e a própria assistência a ser prestada (PINHEIRO, COUTO e NOGUEIRA 2012).

É possível observar que os serviços de saúde ainda não estão organizados para receber esse usuário e atende-lo diante suas necessidades. O homem precisa sentir-se acolhido nesses serviços ficando mais a vontade para expor suas demandas de cuidados e, consequentemente, perceber-se como participativo e inserido nos serviços de saúde. Em compensação, é imprescindível que os profissionais sejam bem preparados para atender esse público alvo e para uma melhor formação profissional, contudo, é necessário acoplar mudanças nos aspectos gerenciais $\mathrm{e}$ organizacionais dos serviços prestados, bem como nas estruturas físicas, com a finalidade de articular avanços tecnológicos, acolhimento, melhores condições de trabalho e processos 
comunicacionais (CAPRARA; FRANCO, 2006).

A importância de uma comunicação eficaz entre onfermeiro e paciente crítico do sexo masculino

A comunicação é tida como função vital e por meio dela, indivíduos e organização de saúde se relacionam entre si, com o meio ambiente e com as próprias partes do seu grupo, influenciando-se mutuamente e transformando fatos em informações (SPAGNUOLO; PEREIRA, 2007). O processo de comunicação configura- se como uma forma de compreender e compartilhar mensagens enviadas e recebidas, exercendo influência no comportamento das pessoas envolvidas (STEFANELLI; CARVALHO, 2005).

Portanto, a comunicação é uma ação de competência vivenciada no cotidiano dos profissionais de saúde, sendo primordial na avaliação dos cuidados a serem prestados aos pacientes, principalmente na enfermagem (TEIXEIRA, BRAGA; ESTEVES, 2004). Esta competência tornase imprescindível em todas as ações realizadas pela enfermagem, influenciando diretamente na interação, na criação de vinculo, na confiança com o paciente, na convivência com outras pessoas, na adaptação ao que não pode ser mudado, na superação dos bloqueios e nos desafios para enfrentar seus problemas.

Para o enfermeiro, no processo de hospitalização, é fundamental que ele proporcione um novo modo de olhar e agir em relação aos pacientes que estão sob seus cuidados. Quando isso não acontece, comprova-se a ineficácia da comunicação da equipe de enfermagem no âmbito hospitalar (TEIXEIRA, BRAGA e ESTEVES, 2004; MORAIS et al, 2009).

No ambiente nosocomial o enfermo permanece fora do seu ambiente familiar, sendo exposto a um novo estilo de vida, completamente estranho, em que rotinas e normas controlam e determinam suas ações e sua forma de agir, que nem sempre é considerado pelos profissionais que o atendem. Os instrumentos básicos da relação de ajuda, tais como o diálogo e a empatia podem ajudar a diminuir os problemas apresentados pela pessoa hospitalizada, mas como não são trabalhados diariamente pelos profissionais, tem como consequência falhas na comunicação entre a equipe e o paciente (MACHADO, LEITÃO; HOLANDO, 2005).

O enfermeiro deve utilizar a comunicação como um instrumento para humanizar o cuidado, dialogando com o paciente a fim de esclarecer dúvidas quanto aos procedimentos a serem realizados nele, exames diagnósticos, tratamento, ou minimizando suas dúvidas, anseios e angustias causados pela sua condição passiva imposta pela enfermidade e pela hospitalização (MORAIS et al, 2009). Para obter uma maior percepção acerca do processo comunicativo, não como um 
simples recurso teórico, mas com finalidades práticas e educativas, a comunicação efetiva e eficaz corriqueira pode ajudar na conduta dos enfermeiros, bem como assegurar possibilidades terapêuticas na sua atuação, além do desempenho humanista e direcionado ao bem estar daqueles que estão sob seus cuidados (MACHADO, LEITÃO; HOLANDO, 2005).

\section{CONCLUSÃO}

A fim de atender ao anseio de investigar a importância e os fatores que influenciam na comunicação dos enfermeiros com os pacientes críticos do sexo masculino, este estudo viabilizou momentos frutuosos com ascensão de ideias, mas também muitas situações de inquietações devido à escolha dessa temática, pois algumas dificuldades foram encontradas, principalmente no que diz respeito ao escasso material teórico. Contudo, espera-se que esse estudo seja motivador para novas pesquisas na área.

\section{REFERÊNCIAS}

BRASIL. Ministério da Saúde. Secretaria de Atenção a Saúde. Departamento de Ações Programáticas Estratégicas. Política Nacional de Atenção Integral à Saúde do Homem: Princípios e diretrizes. Brasília, DF, 2009. Disponível em: http://portal.saude.gov.br/portal/arquivos/pdf/politica nacional_homem.pdf. Acesso em 02 jul 2013.
A partir desta pesquisa foi possível inferir que o homem encontra empecilhos, medo e insegurança em está em um serviço de saúde, não conseguindo separar o seu lado dominador, viril, forte e invulnerável.

Neste sentido, foi possível observar que os fatores que influenciam na comunicação do paciente crítico do sexo masculino são as questões relacionadas ao gênero, a masculinidade e a cultura. Sendo necessária elencar novas estratégias de comunicação para repassar aos pacientes críticos do sexo masculino, por ser um público em que é preciso ter mais cautela, saber abordar, dialogar e repassar as informações e os procedimentos que serão realizados.

Desmistificar as visões constituídas pela sociedade, não é tarefa fácil é necessário da colaboração de todos os profissionais. A masculinidade ainda é algo que impede os homens de sentir- se dependentes de cuidados ofertados pelo enfermeiro, em que a comunicação é por vezes a forma de demonstração dessa atitude assumida tanto pelos profissionais quanto pelos pacientes.

CAPRARA, A.; FRANCO, A.L.S. Relação médico paciente e humanização dos cuidados em saúde: limites, possibilidades e falácias. In: DESLANDES, S.F. (Org.). Humanização dos cuidados em saúde: conceitos, dilemas e praticas. Rio de Janeiro: Fiocruz, 2006. p. 85-108.

COURTENAY, W. H. Construction of masculinity and their inluence on men's well- being: a theory of gender and health. Soc. Sci. Med. v.50, n.10, p.1385401,2000 
COSTA, K. N. F. M. Modelo de comunicação verbal com o cego: desenvolvimento e validação em consulta de enfermagem [tese-doutorado]. Fortaleza (CE): Universidade Federal do Ceará; 2009.

COUTO, M. T.; MACHIN, R. A produção dos corpos masculinos como lócus do cuidado: uma análise de gênero acerca dos profissionais da saúde. Psicologia, Conocimiento y Sociedad 2 (2), 84- 105 (Nov. 2012) Trabajos Originales.

GOMES, R et al. Os homens não vêm! Ausência e/ou invisibilidade masculina na atenção primária. Ciência \& Saúde Coletiva, v.16 (Supl. 1) p.983-992, 2011.

GOMES, R.; NASCIMENTO, E.F. A produção do conhecimento da saúde pública sobre a relação homem-saúde: uma revisão bibliográfica. Cad. Saude Publica, v.22, n.5, p.901-11, 2006.

LAKATOS, E. M.; MARONI, M. A. Metodologia Cientifica- Ciência e conhecimento cientifico, métodos científicos, teoria, hipótese e variáveis. 6 ed. São Paulo: Atlas.

MACHADO, M. M. T.; LEITÃO, G. C. M.; HOLANDA, FUX. O conceito de ação comunicativa: uma contribuição para a consulta de enfermagem. Rev Lat Am Enfermagem. 2005;13(5): 723-28.

MORAIS, G. S. N.; COSTA, S. F. G.; FONTES, W. D, CARNEIRO, A. D. Comunicação como instrumento básico no cuidar humanizado em enfermagem ao paciente hospitalizado. Acta paul. enferm. 2009;22(3):54-63.

NASCIMENTO, A. R. A.; TRINDADE, Z. A.; GIANORDOLI-NASCIMENTO, I. F.; PEREIRA, F. B.; SILVA, S. A. T. C.; CERELlO, A. C. Masculinidades e Práticas de Saúde na Região Metropolitana de Belo Horizonte - MG. Saúde Soc. São Paulo, v.20, n.1, p.182-194, 2011

PINHEIRO, T. F; COUTO, M. T; y NOGUEIRA, da S. G. (2012) Homens e cuidado: Construções de masculinidades na saúde pública brasileira.

Psicología, Conocimiento y Sociedad, 2(2), 177-195. Acesso em: 05/02/2014. Disponível em: a e://revista.psico.edu.uy/ a ex.php/revpsicologia /article/view/142/78

SILVA, F. C. B. da.; BRITO, R. S. de. Percepção de gestantes acerca das atitudes do companheiro diante da sua ausência no pré- natal. Rev. Rene. Fortaleza, v. 11, n. 3. p. 95-102, jul./set.2010. Acesso em: 12 jun 2013. Disponível em: http://www.revistarene.ufc.br/revista/index.php/revista /article/view/400/pdf

SILVEIRA, S. A. S.; BARBOSA, R. F. A participação masculina no planejamento familiar no programa de saúde da família em Campina Grande- PB. 2009. Disponível em: http: // www.itaporanga.net/genero/1/GT06/14.pdf. Acesso em: 03/01/2014.

SPAGNUOLO, R. S.; PEREIRA, M. L. T. Práticas de Saúde em Enfermagem e Comunicação: um estudo de revisão da literatura. Ciênc Saúde Colet [Internet]. 2007

STEFANELli, M. C.; CARVALHO, A. (Org). A comunicação nos diferentes contextos da enfermagem. Barueri (SP): Manole; 2005.

TEIXEIRA, A. L.; BRAGA, A.; ESTEVES, M. C. A comunicação com a criança na punção venosa: percepção do enfermeiro. Revista do Hospital de Crianças Maria Pia. 2004;13(3):187-94.

Rafaella Alves Sarmento Costa

Residente em Saúde da Família em Comunidade pela Faculdade de Ciências Médicas da Paraíba

Camila Carla Dantas Soares

Mestre pela Universidade Federal da Paraíba

Jeferson Barbosa Silva

Dourando pela Universidade Federal da Paraíba

Jocelly de Araújo Ferreira
Doutoranda pela Universidade Federal de Minas
Gerais

